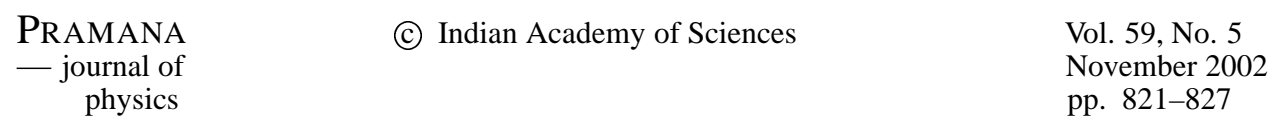

\title{
ANU LINAC upgrade using multi-stub resonators
}

\author{
D C WEISSER and N R LOBANOV \\ Department of Nuclear Physics, Research School of Physical Sciences and Engineering, \\ Australian National University, Canberra, ACT 0200, Australia
}

\begin{abstract}
A proposal has been prepared to upgrade the LINAC at ANU, using re-plated PbSn split loop resonators performing at $3.6 \mathrm{MV} / \mathrm{m}$, and the addition of two- and three-stub resonators. The system is designed to provide $6 \mathrm{MeV} / \mathrm{a} . \mathrm{m} . \mathrm{u} .{ }^{107} \mathrm{Ag}$ starting with gas-stripped beam from the $14 \mathrm{UD}$, which is then foil-stripped before the LINAC. No major changes to the beam optics components will be required other than addition of a large bore rebuncher in the middle of the $180^{\circ}$ achromat. Models of the two- and three-stub resonators have been built and optimized for the goal frequency, for the separation of accelerating and other modes as well as for the minimization of the current in the demountable joints. A copper prototype has been constructed and is being plated with PbSn for cold testing.
\end{abstract}

Keywords. Electrostatic; collective and linear accelerators; electrostatic accelerator; LINAC; superconducting resonator.

PACS No. 29.17.+w

\section{Introduction}

LINAC development work at ANU is currently aimed at improving performance and further expanding the existing superconducting heavy-ion accelerator. We used MSA chemistry to re-plate the six split loop resonators (SLRs) obtaining a field gradient of $3.6 \mathrm{MV} / \mathrm{m}$ - a significant improvement over their previous performance of $\sim 1.7 \mathrm{MV} / \mathrm{m}[1,2]$. The LINAC upgrade proposes adding eight $\beta=0.065$ three-stubs resonators, three Applied Superconductivity Inc (ASI) SLRs, eight $\beta=0.1$ two-stub resonators and four $\beta=0.15$ two-stub resonators. The design features of the multi-stub resonators include a demountable stub assembly employing an rf gasket with acceptably low current [3,4]. The fabrication of resonators is greatly simplified by eliminating electron beam welding or vacuum brazing. All cavities for the ANU LINAC operate at a frequency of $150 \mathrm{MHz}$. The new geometries are consistent with either $\mathrm{Nb}$ sputter-coating or lead plating onto copper substrates. The upgraded cryogenic system, based on a Sultzer TCF50 Cold Box operating at $328 \mathrm{~W}$, has sufficient capacity to service future cryostats. With a nominal $6 \mathrm{~W}$ rf load in each of the 11 resonators installed in 5 cryostats, there is about $180 \mathrm{~W}$ excess cooling capacity. The cooling power can be further increased with liquid nitrogen pre-cooling to the cold box. This is yet to be exercised. 


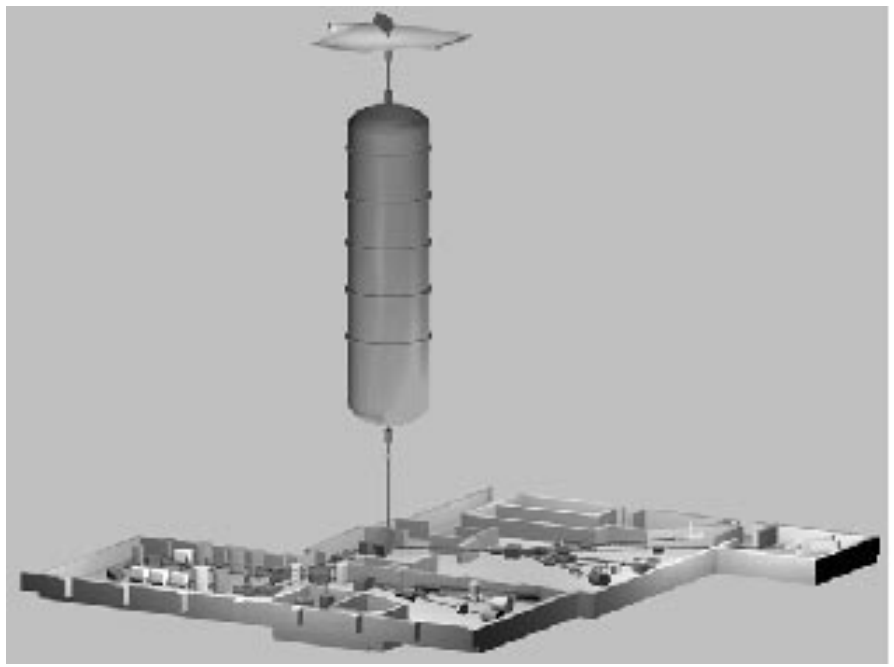

Figure 1. ANU heavy-ion accelerator facility.

\section{ANU LINAC upgrade using multi-stub resonators}

All new technologies required to implement the project are available at ANU immediately. Figure 1 shows the layout of the proposed facility. The LINAC at present comprises nine $\beta=0.1$ SLRs housed in three ASI module cryostats. Before entering the LINAC, the nanosecond pulsed beam is further compressed to $\sim 100 \mathrm{ps}$ width by a superconducting quarter wave resonator, QWR. A similar QWR is used in time-energy lens which either rebunches or homogenize the energy of the beam leaving the LINAC. There is room in the first leg for two additional cryostats, shown housing eight three-stub $\beta=0.065$ resonators. The cryostats for these resonators are similar to those designed at the Weizmann Institute and manufactured by Janis Co. [5]. The accelerated beam traverses a $180^{\circ}$ achromat and is then directed along an as yet unpopulated line. This line will accommodate one additional ASI module cryostat with three $\beta=0.1$ lead-plated SLRs. Until then, it will serve three Janis-like cryostats with eight $\beta=0.1$ two-stub resonators and four $\beta=0.15$ two-stub resonators. The superconducting booster is designed to provide $6 \mathrm{MeV} / \mathrm{a} . \mathrm{m} . \mathrm{u} .{ }^{107} \mathrm{Ag}$ starting with gas stripped beam from $14 \mathrm{UD}$, which is foil stripped before the LINAC. Employment of the LINAC foil stripper extends the range of the $180^{\circ}$ achromat $\left(M E / Q^{2}=123\right)$ for beams with $M$ up to 200 , though at the cost of beam intensity. No major changes to the beam optics components will be required other than the addition of a large bore rebuncher in the middle of the $180^{\circ}$ achromat in order to reduce the bunch time spread due to the long drift space between the two legs of the LINAC.

\section{Progress in multi-stub resonators modeling}

\subsection{Two-stub resonator - mode splitting and tuning}

The half wave resonator structure is inspired by the Delayen design [6]. It can be also thought of as a straight inductor version of an SLR (figure 2). The center column has a 

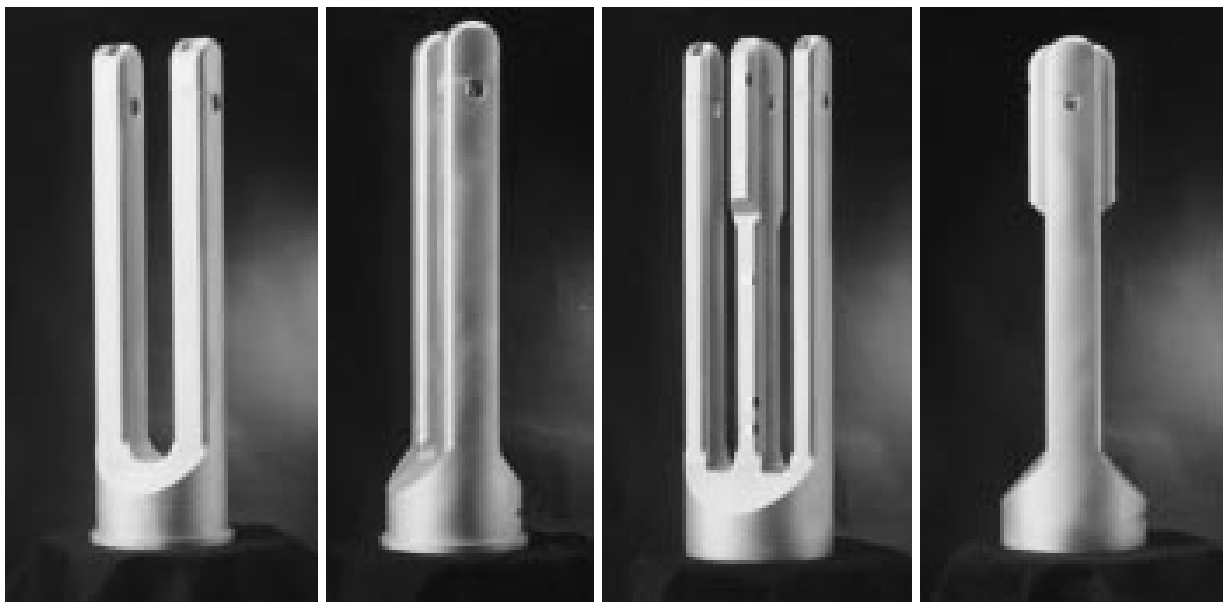

Figure 2. Two-stub and three-stub resonators.

diameter of $16 \mathrm{~cm}$ at the shorted end and splits to two stubs with average diameter of $6 \mathrm{~cm}$. The current needed to establish the high voltage on the drift tubes is carried between the stubs in a balanced inductive loop. The two-stub resonator has two principal low frequency eigenmodes, the accelerating mode in which charge oscillates between the stubs and a quarter wave mode in which charge oscillates between the pair of stubs in parallel and the outer wall. The efficiency of the accelerating mode is maximum when the gap between drift tubes is approximately twice the end gap [7]. Imbalance in the stub configuration can produce coupling between the accelerating and the quarter wave modes, increasing the rf current through the rf joint at the base of the outer wall and so increasing joint losses. The design shown in figure 2 minimizes this current by shifting the quarter wave mode to a lower frequency - an effect strongly dependent on the length of the central column $L_{\mathrm{c}}$. For example, mode splitting is $12.1 \mathrm{MHz}$ for $L_{\mathrm{c}}=9 \mathrm{~cm}$ and $17.4 \mathrm{MHz}$ for $L_{\mathrm{c}}=15 \mathrm{~cm}$.

The stub assembly is mounted in a $19.4 \mathrm{~cm}$ diameter cylinder, $60 \mathrm{~cm}$ long and closed with the tuner plate. The distance between tuner plate and inner conductor $L_{\text {tun }}$ of about 5 $\mathrm{cm}$ was chosen for a tuning sensitivity of $8 \mathrm{kHz} / \mathrm{mm}$. Straight rather than tapered inductors are expected to minimize multipactoring and, together with a curved shorting plate, are good for surface preparation and coating.

\subsection{Three-stub resonator - mode splitting and tuning}

The three-stub resonator (figure 2) has three eigenmodes:

- the accelerating mode in which charge oscillates between the outer stubs and the central one;

- the half wave mode in which charge oscillates between two outer stubs by-passing the central one;

- the quarter wave mode in which charge oscillates in parallel in all stubs relative to the outer wall. 

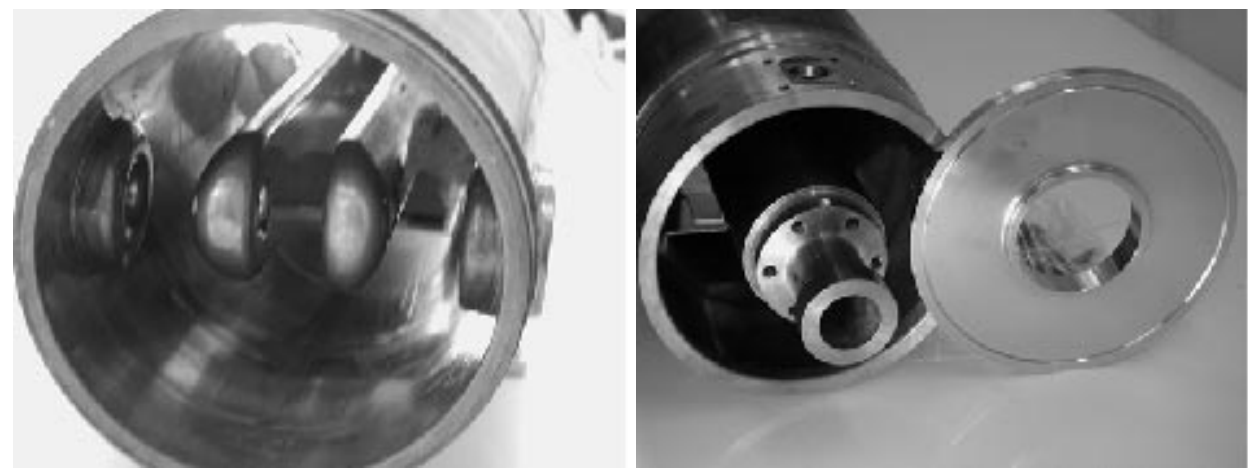

Figure 3. Copper prototype for $\beta=0.1,150 \mathrm{MHz}$ two-stub resonator using two $\mathrm{rf}$ demountable joints between the stub assembly and outer wall. Only one joint will be used in production resonators.

As in the two-stub resonator, the efficiency of the accelerating mode is maximum when the interstub gaps are approximately twice the end gaps [7]. With this spacing, the electric forces are balanced minimizing distortion of the structure [7]. The frequency splitting between the accelerating and quarter wave modes is controlled by the length of the central mount $L_{\mathrm{c}}$ as for the two-stub design. For example, the non-accelerating mode is shifted by 14.9 MHz for $L_{\mathrm{c}}=9 \mathrm{~cm}$ and by $19.3 \mathrm{MHz}$ for $L_{\mathrm{c}}=15 \mathrm{~cm}$. The balance of field profile, mirror currents in the walls and induced charge on the tuner plate will be achieved by selective machining of the inductors. For a tuner plate to inner conductor distance, $L_{\text {tun }}$, of about $5 \mathrm{~cm}$, a tuning range of $10 \mathrm{kHz} / \mathrm{mm}$ was measured.

\section{Electromagnetic properties}

A conventional series of measurements was performed on the resonators at room temperature including measurement of the $Q_{0}$ to obtain the shunt impedance. A bead test was used to obtain the field profile, the accelerating field and energy content, and the transit time factor. Extensive room temperature tests were performed on the aluminium model in order to map the magnetic field and to determine mode frequencies and balance.

The stub geometry was developed with a help of Superfish/Poisson software [8]. A set of expressions for the electromagnetic properties of low velocity accelerating structures based on quarter-wavelength resonant lines [9] was used to derive the electromagnetic properties as described in [2]. Aluminium models of the two- and three-stub designs have been built and optimized for frequency, splitting between accelerating and other modes as well as to minimize the current in the demountable joints. A copper prototype (figure 3) has been constructed and is being prepared for plating with $\mathrm{PbSn}$ and $\mathrm{Nb}$ sputtering. Cold testing will be done first with the PbSn-plated version. A prototype for a $\beta=0.1,150 \mathrm{MHz}$ twostub resonator employs the outer can from a $150 \mathrm{MHz}$ QWR, and so this test device has two demountable joints at the shorting plate rather than one in the final design. 


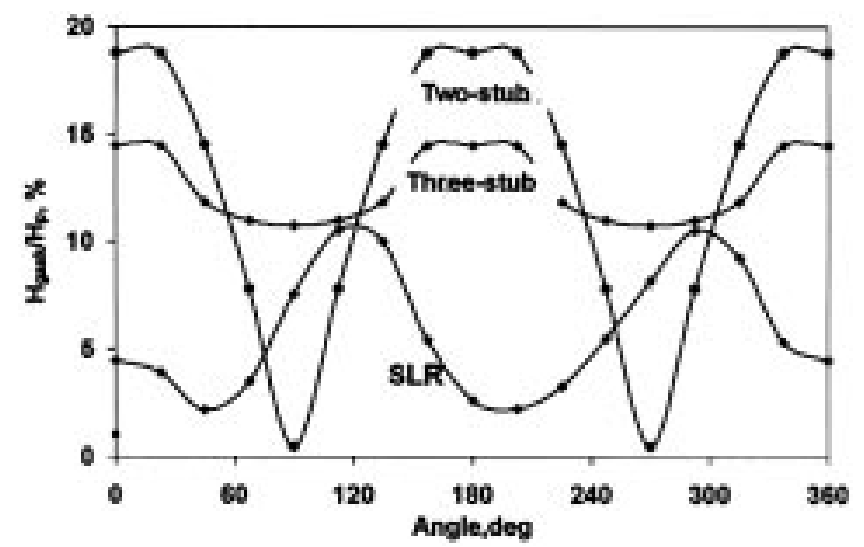

Figure 4. Magnetic field profiles for SLR and multi-stub resonators along the circular rf gasket.

\section{Demountable joints}

The proposed structures have one joint between the outer wall and the shorting plate and one joint between the tuner plate and the outer wall to facilitate construction and coating of the resonator. We wish to eliminate electron beam welds because a convenient facility is not locally available and electron beam welding of copper often results in micro-cracks, craters, hidden voids and material projections. Multi-stub resonators intrinsically impose minimal currents on these joints and our design allows control even over these. Nevertheless, even small currents in poor rf joints cause losses and so low loss joints are desirable.

Low loss demountable joints for $\mathrm{Nb}$ resonators have been developed and are in daily use at ANL and JAERI. These are expensive because of the extremely strong flanges required and of the cost of fabrication of the $\mathrm{Nb}$ gaskets. The less expensive alternatives using lead wire or indium-tin both have given good results in SLRs [7,10]. In [11], thermometry demonstrated that, in the absence of field emission, only about $5 \%$ of the total $4.2 \mathrm{~K}$ losses occur on the SLR's outer housing. rf Power losses in a lead gasket with surface resistance $R_{\mathrm{S}}$ is given by $P_{\text {gask }}=0.5 R_{\mathrm{S}}\langle H\rangle^{2} \pi D_{\text {gask }} t$ where $\langle H\rangle$ is the average magnetic field at the joint, $D_{\text {gask }}$ and $t$ are the major diameter and thickness of the gasket respectively. For different cavities with comparable peak magnetic fields $H_{\mathrm{p}}$, the rf losses in the gasket are proportional to the dimensionless parameter $P_{\text {gask }} \sim\left(\langle H\rangle / H_{\mathrm{p}}\right)^{2} D_{\text {gask }} \lambda^{-1}$, where $\lambda \approx 2$ $\mathrm{m}$ is the free space wavelength. The ratio $\langle H\rangle / H_{\mathrm{p}}$ can be determined experimentally by mapping magnetic field along the gasket using an induction loop probe (figure 4).

The data presented in figure 4 are for a column length, $L_{\mathrm{c}}$, of $10 \mathrm{~cm}$ in two-stub and three-stub resonators. The design had not yet been optimized to match mirror currents in the wall. For $L_{\mathrm{c}}=10 \mathrm{~cm}$, the maximum value of $H_{\text {gask }} / H_{\mathrm{p}}$ for a three-stub resonator is 1.8 and two-stub resonator is 1.4 compared to a unity for the SLR. However, each gasket in the SLR is twice as long as the single gasket in the new resonator. Thus rf losses in the gasket of the new resonator before optimization are half of that in SLRs. Comparative losses in rf joint of SLR and multi-stub resonators were $P_{\mathrm{SLR}}: P_{\text {three-stub }}: P_{\text {two-stub }}=1.8: 1.0: 1.02$. These losses could be reduced even further by increasing $L_{\mathrm{c}}$ and adjusting the coupling 

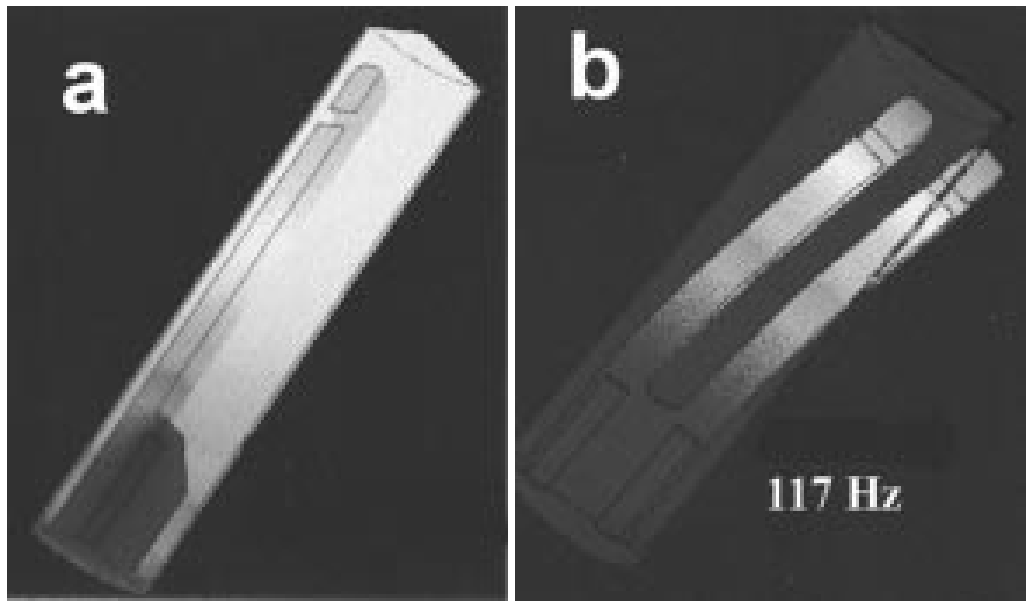

Figure 5. One quarter of the model cut along the symmetry planes is shown: (a) the temperature profile and (b), the lowest vibrational mode of the two-stub resonator. The temperature increase in the light areas is $0.4 \mathrm{~K}$.

between the stubs relative to the coupling to the wall. The losses reported here are only for the stub assembly to wall joint. There will be additional losses in the tuner plate to wall joint which is not present for the SLR.

\section{Mechanical stability and temperature stabilization}

A detailed account of thermal and structural modeling of two-stub and three-stub resonators is given in [12]. Temperature and mechanical characteristics of multi-stub resonators were simulated using finite element software 'Pro/Engineer' [13]. Figure 5a illustrates the variation in the temperature profile at $6 \mathrm{~W}$ absorbed rf power and $5 \mathrm{~b}$ illustrates the lowest mechanical vibrational mode of the two-stub resonator. The interface between the liquid helium and the stub assembly has a length of $14 \mathrm{~cm}$ and diameter of $1.5 \mathrm{~cm}$. The model shows components on one side of each symmetry plane of the two-stub resonator. Thus only one stub is illustrated. The lowest mechanical vibration frequency is $117 \mathrm{~Hz}$ and the maximum temperature increase is $0.4 \mathrm{~K}$. Increasing the thickness of the stubs would raise the frequency beyond this already acceptable level.

A small displacement of the drift tube along the beam axis corresponding to the mechanical mode shown in figure $5 \mathrm{~b}$ will result in a net electric force known as the radiation pressure frequency shift $\Delta f=-k E_{\mathrm{a}}^{2}$. This effect is very strong in SLRs where $k \approx 100$. In multi-stub resonators, the radiation frequency shift is expected to be similar to the mechanically stable QWR structures, $k \approx 0.4-0.6[10,11]$.

\section{Conclusions}

A road map has been drawn for the continuing upgrade of the ANU LINAC. It will exploit either proven $\mathrm{PbSn}$ plating or $\mathrm{Nb}$ sputtering and multi-stub resonators. The $\mathrm{PbSn}$ plating chemistry, which has provided 3.6 MV/m in SLRs, needs to be demonstrated in multi-stub 
resonators. Further, $\mathrm{Nb}$ sputtering of these complex shapes needs to be explored. Measured characteristics for three-stub and two-stub resonators encourage their use. To confirm cavity parameters, prototypes will be cold tested in 2002. Other areas of LINAC infrastructure development include demonstrating that the cryogenic system has the capacity to serve future cryostats and modernization of the vacuum instrumentation. The LINAC's modularity allows development as resources permit, concurrent with the exploitation of its increasing capabilities for nuclear physics research.

\section{Acknowledgement}

The authors would like to thank their colleagues from ANL, Stony Brook and INFN for many useful and clarifying discussions.

\section{References}

[1] N R Lobanov and D C Weisser, Proc. 9th Workshop on RF Superconductivity edited by A Robinson and B Rusnak, 1999, Santa Fe, pp. 129-142

[2] N R Lobanov and D C Weisser, Proc. 10th Workshop on RF Superconductivity, 2001, Tzukuba, Japan (to be published)

[3] N R Lobanov and D C Weisser, Proc. 31st SNEAP, Knoxville, October 1999 (to be published)

[4] N R Lobanov and D C Weisser, Proc. 9th Workshop on RF Superconductivity edited by A Robinson and B Rusnak, 1999, Santa Fe, pp. 124-128

[5] I Ben-Zvi, B V Elkonin, J S Sokolovsky and I Tserruya, Nucl. Instrum. Methods 244, 306 (1986)

[6] J R Delayen and J E Mercereau, Nucl. Instrum. Methods Phys. Res. A257, 71 (1987)

[7] J R Delayen, G J Dick and J Mercereau, IEEE transactions on magnetics MAG-17, 939-942 (1981)

[8] J H Billen and L M Young, Proc. 1993 Particle Accelerator Conference, Vol. 2 of 5, p. 790

[9] I Ben-Zvi and J M Brennan, Nucl. Instrum. Methods 212, 73 (1983)

[10] J Noe, Proc. HIAT98 edited by K W Shepard, American Institute of Physics Proceedings 473, 1999, pp. 192-213

[11] J W Noe, J Rico, H Uto and T Wada, Proc. 6th Workshop on RF Superconductivity edited by R Sundelin, 1993, pp. 1052-1064

[12] N R Lobanov, D Weisser, S Kalyanasundaram and J M Donnelly, Proc. 10th Workshop on rf Superconductivity, 2001, Tzukuba, Japan (to be published)

[13] R Toogood, Pro/engineer tutorial, SDC publications, 1999 